E9-2011-84

Bing Wang ${ }^{1}$, Huanfeng $\mathrm{Hao}^{1}$, S. B. Vorozhtsov, V. L. Smirnov, Qinggao Yao ${ }^{1}$, Jinquan Zhang ${ }^{1}$, Mingtao Song ${ }^{1}$, Hongwei Zhao

\title{
COMPUTER DESIGN OF A COMPACT CYCLOTRON
}

Submitted to «Письма в ЭЧАЯ»

${ }^{1}$ Institute of Modern Physics, Lanzhou, China 
Ван Б. и др.

Компьютерное моделирование компактного циклотрона

Представлены результаты компьютерного моделирования структурных элементов компактного циклотрона на примере инжектора циклотрона HITFiL, coздаваемого в Институте современной физики (Ланьчжоу, Китай). В статье описан комплексный подход к моделированию компактного циклотрона, включающий расчет электромагнитных полей структурных элементов и расчет динамики пучка. Предложены оптимальные параметры структурных элементов установки, внесены предложения по изменению существующего начального технического проекта установки, что привело к заметному повышению качества и трансмиссии пучка.

Работа выполнена в Лаборатории ядерных проблем им. В.П. Джелепова ОИЯИ.

Препринт Объединенного института ядерных исследований. Дубна, 2011

Bing Wang et al.

E9-2011-84

Computer Design of a Compact Cyclotron

Here we present results of the computer design of the structural elements of a compact cyclotron by the example of HITFiL cyclotron selected as the driving accelerator that is under construction at the Institute of Modern Physics (Lanzhou, China). In the article a complex approach to modeling of the compact cyclotron, including calculation of electromagnetic fields of the structural elements and beam dynamics calculations, is described. The existing design data on the axial injection, magnetic, acceleration and extraction systems of the cyclotron are used as a starting point in the simulation. Some of the upgrades of the cyclotron structural elements were proposed, which led to substantial improvement of the beam quality and transmission.

The investigation has been performed at the Dzhelepov Laboratory of Nuclear Problems, JINR. 


\section{INTRODUCTION}

Nowadays, the cyclotron design requires an intensive and detailed computer simulation. An example was a design of the new accelerator complex HITFiL (Heavy Ion Therapy Facility in Lanzhou) that is under construction at the Institute of Modern Physics (IMP), Lanzhou [1]. In this project, a $7 \mathrm{MeV} / \mathrm{u}{ }^{12} \mathrm{C}^{5+}$ cyclotron (Fig. 1) is selected as the driving accelerator providing the $10 e \cdot \mu \mathrm{A}$, $\varepsilon=20 \pi \cdot \mathrm{mm} \cdot \mathrm{mrad}, \Delta W / W \leqslant \pm 1 \%$ carbon beam [2]. It is conceived as an injector for the HITFiL synchrotron, which accelerates carbon ions to the energy $400 \mathrm{MeV} / \mathrm{u}$ for tumor treatment.

In the previous design of the cyclotron [2] (Technical Design Baseline, TDB) there were some drawbacks which prevented reaching the required beam quality and intensity. The most important are as follows: lower bunching efficiency in the axial injection system, crossing of dangerous resonances in the acceleration region, missing focusing elements in the extraction system, etc.

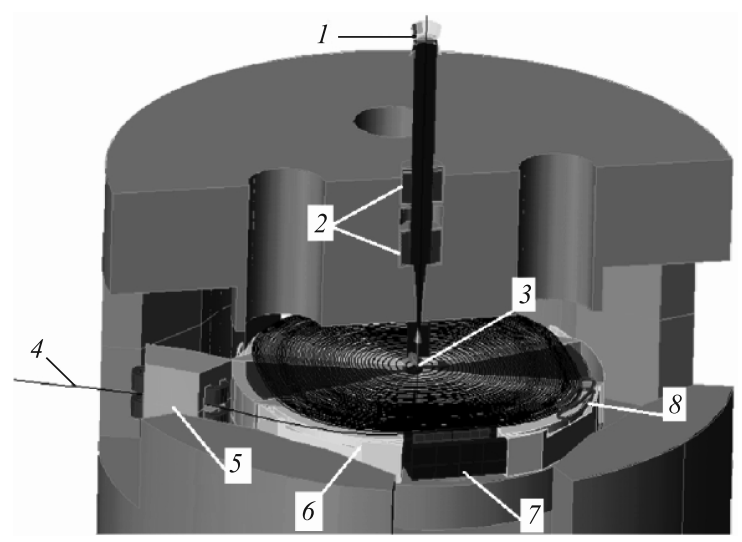

Fig. 1. Computer model of the HITFiL cyclotron: $1-$ buncher; $2-$ solenoids; $3-$ inflector; 4 - reference particle trajectory; 5 - magnetic channel (M2); 6 - magnet sector; 7 - electrostatic deflector (ES1); 8 - harmonic coil 
To overcome the TDB problems and to obtain the required cyclotron beam intensity and quality, the following approach was considered in this work:

1. Generation of the three-dimensional electric and magnetic field maps where they were appropriate or missing.

2. Analysis of the performance of the cyclotron units: LEBT (Low Energy Beam Transport), CR (Central Region), Acceleration, Extraction, Beam Delivery System (BDS) to deliver beam to the HEBT (High Energy Beam Transport).

3. Estimation of the overall cyclotron transmission (exit-to-entrance beam intensity ratio) and output beam parameters (emittance, energy spread, time pulse, and dispersion).

The aim of the simulation was to increase the cyclotron transmission and output beam quality by improving the matching at the injection, optimizing the acceleration regime parameters, and increasing focusing at the extraction. The existing TDB data on the axial injection, magnetic, acceleration and extraction systems of the cyclotron [2] are used as a starting point in the calculation.

The three-dimensional electric and magnetic field maps used in the beam dynamics analysis were calculated with the VF Opera3D software [3]. Generation of the $3 \mathrm{D}$ computer model of the RF system including the surrounding ground and inflector was performed in the course of the study (Fig. 2). The geometrical model based on the IMP input data was prepared first. The combined layout of the RF and magnetic system was made.

The magnetic field of the cyclotron was also calculated with Tosca [3] using the already existing IMP model (Fig. 3). Assessment of the simulation accuracy by changing the number of FEs from 0.6 million to 3 million was performed.

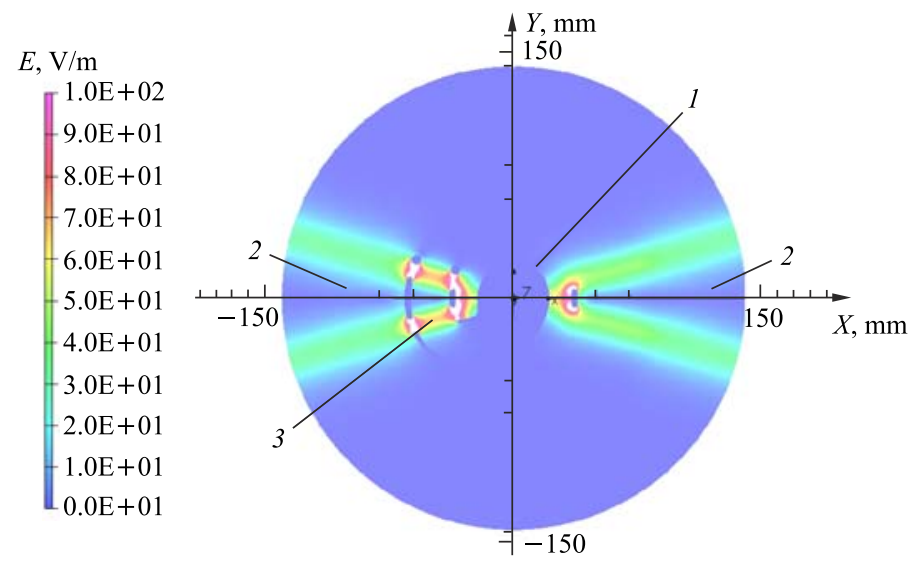

Fig. 2. Strength of the electrical field distribution in the cyclotron central region: 1 inflector cage; 2 - dee; 3 - first acceleration gap 


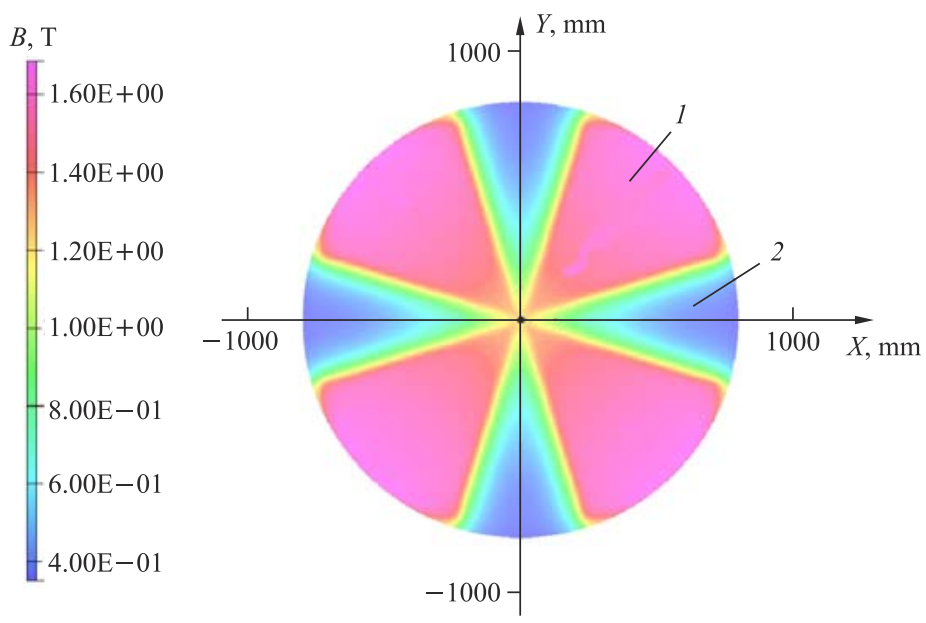

Fig. 3. Magnetic field distribution: $1-$ sector region; $2-$ valley region

Along with the good agreement of the results, obtained with different meshing, there is still some field distribution uncertainty in the central region of the machine that will be removed by the magnetic field measurements.

For the analysis of the beam dynamics a detailed computer model of the cyclotron including all structural elements from the buncher to the first HEBT lens was created (Fig. 1). The emphasis in the calculations was placed on the extraction system. Figure 4 shows the cyclotron layout with the extraction system elements.

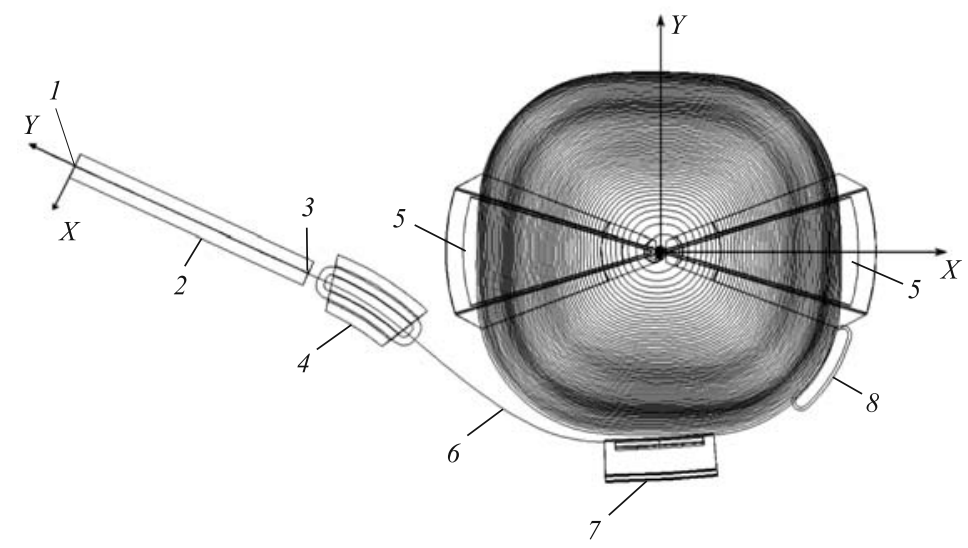

Fig. 4. Cyclotron layout: $1-1$ st HEBT lens; $2-$ BDS; 3 - IP (Interface Point); $4-$ magnetic channel; $5-$ dee; 6 - reference particle trajectory; 7 - electrostatic deflector; 8 - harmonic coil 
Table 1. Basic design parameters

\begin{tabular}{|l|c|}
\hline \multicolumn{1}{|c|}{ Parameter } & Value \\
\hline Center magnetic field, T & 1.2128 \\
Injection radius, cm & 2.7 \\
Injection energy, keV & 111.6 \\
RF frequency, MHz & 31.023 \\
Harmonic number & 4 \\
Extraction radius, cm & 75 \\
\hline
\end{tabular}

The basic design parameters of the cyclotron are given in Table 1.

\section{BETATRON TUNES}

Analysis of the cyclotron magnetic field, corresponding to the latest version of the TDB, shows that there are crossings of the dangerous resonances in the acceleration process (Fig. 5, solid line). Frequencies of the betatron oscillations were calculated for a set of specified energies by the well-known equilibrium-orbit code CYCLOPS [4].

A modification of the magnet pole structure was proposed to eliminate the problem. Along with some change of the magnetic sector shape, the air gap

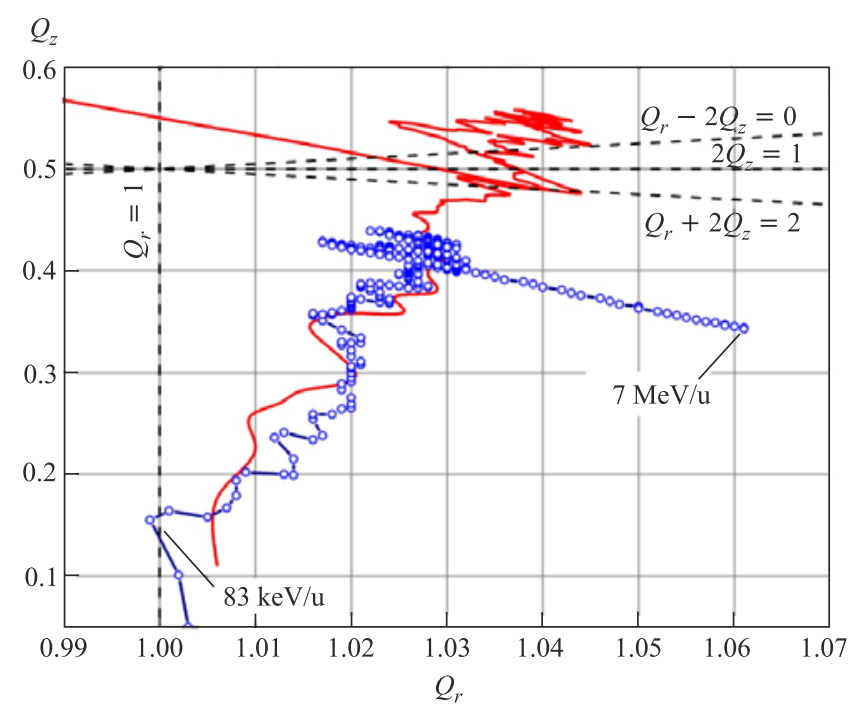

Fig. 5. Tune diagram for two magnet structures with different air gaps between the sectors: $50 \mathrm{~mm}$ (solid line) and $80 \mathrm{~mm}$ (circles) 
between the sectors was increased to $80 \mathrm{~mm}$ instead of $50 \mathrm{~mm}$ in the previous design. In the new magnetic field there is no crossing of dangerous resonances except the integral $Q_{r}=1$ resonance at the 1 st turn $(R=80 \mathrm{~mm})$ in the cyclotron (Fig. 5, circles). The beam dynamics calculations with the first imperfection harmonic of reasonable amplitude $\sim 1 \mathrm{mT}$ show that there is practically no deterioration of the radial beam quality due to this resonance since the ions are accelerated through it very fast.

\section{BUNCHER}

On the basis of the assessment of the magnetic and electrostatic fields of the axial injection line structure elements, the particle tracking from the buncher entrance to the midplane of the cyclotron was performed. In calculations of the buncher performance the newly developed SNOP code was used. It was assumed that the axial position of the buncher is $Z=1300 \mathrm{~mm}$, the gap with the uniform $\mathrm{RF}$ field is $5 \mathrm{~mm}$, and the incident ion bunch is of $360^{\circ} \mathrm{RF}$ phase angle. The initial transverse emittance at the buncher entrance corresponded to that of the ECR ion source output and was varied in the range $75-150 \pi \cdot \mathrm{mm} \cdot \mathrm{mrad}$ to see the effect of the transverse quality of the injected beam on the performance of the cyclotron units. The initial 10000 particle distribution upstream of the buncher with the transverse emittance $125 \pi \cdot \mathrm{mm} \cdot \mathrm{mrad}$ and the injection energy $111.6 \mathrm{keV}$ was used in the calculation. It was assumed that there is no buncher fringe field at this location with the buncher. The spiral inflector was off, and its impact on the transmission will be considered in the following section devoted to the central region simulations.

The optimal buncher voltage according to the classical PIC [5] method (FFT, $\sim 10^{5}$ nodes of the Cartesian coordinate mesh) for the beam space charge (SC) effect calculations varies almost linearly in the range $290-330 \mathrm{~V}$ for the incident beam intensity between 0 and $200 e \cdot \mu \mathrm{A}$. The results somewhat agree with the previously published ones, see [6]. The SC effects apparently reduce the bunching efficiency (the ratio of the ions in the RF phase range $\pm 10^{\circ}$ at the midplane to the total number of ions in the incident $\mathrm{CW}$ beam) from $\sim 41.5$ to $\sim 39 \%$ at the nominal beam intensity $200 e \cdot \mu \mathrm{A}$. Figure 6 shows relative particle energy dependence on the RF phase in the median plane for the sine-wave buncher adopted in the TDB case.

The bunching efficiency could be substantially increased with the saw-tooth buncher design. The buncher structure from [7] was adopted for the calculations (Fig. 7). This buncher has the $87 \%$ transparency grid and $7 \mathrm{~mm}$ gap between the RF electrodes similar to the RIKEN AVF cyclotron [8].

In the saw-tooth buncher simulations the initial beam started at the buncher entrance and had transverse emittance $75 \pi \cdot \mathrm{mm} \cdot \mathrm{mrad}$. The calculations show that the buncher fringe field leads to some widening of the longitudinal size of 

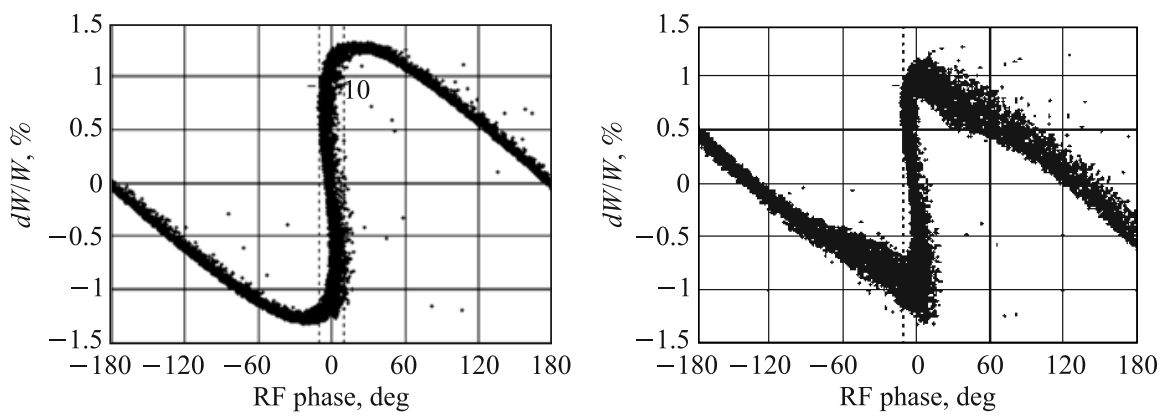

Fig. 6. Midplane longitudinal ion distributions with the incident beam intensity $0 e \cdot \mu \mathrm{A}$ (left) and $200 e \cdot \mu \mathrm{A}$ (right) for the sine-wave buncher

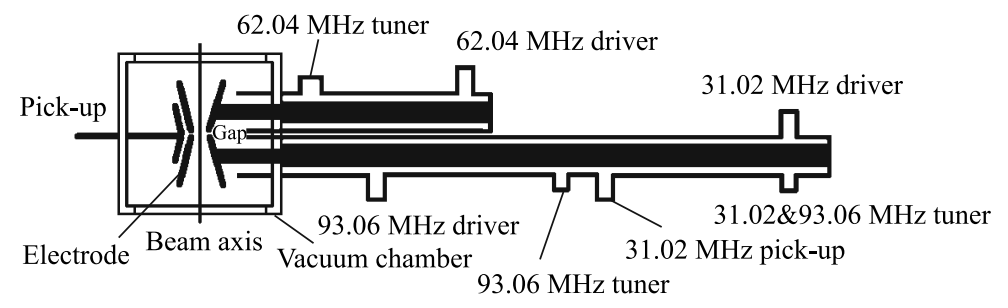

Fig. 7. Initial saw-tooth buncher design
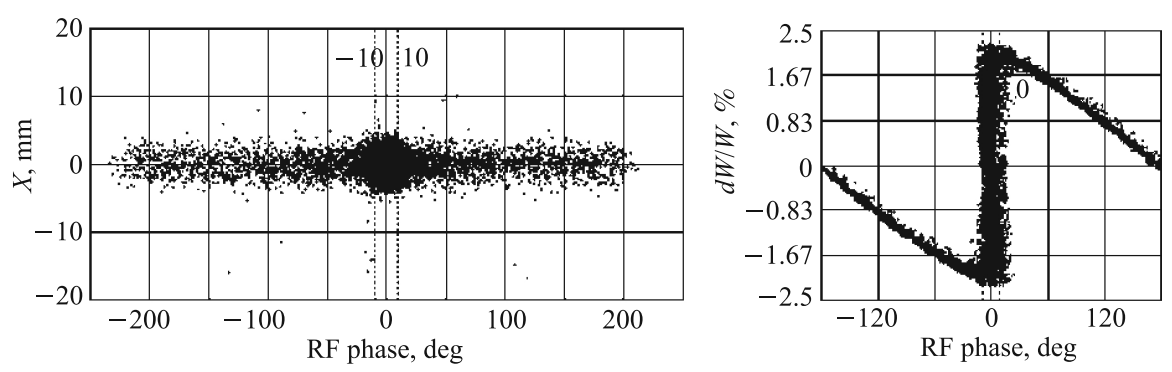

Fig. 8. Saw-tooth buncher impact on the beam at the inflector entrance (left) and midplane (right)

the bunch in the position of the focus. The effect slightly decreases the bunching efficiency. The resulting effect of the saw-tooth buncher on the longitudinal ion motion is seen in Fig. 8, where $\sim 61 \%$ of the particles are found in the $\pm 10^{\circ} \mathrm{RF}$ phase angle range compared with $\sim 41 \%$ for the sine-wave buncher. The SC effects decrease the number by a few percent. These effects were assessed only 
in the LEBT, namely, for the bunching efficiency estimation. Downstream of the CR there is no need to take into account the beam self-field since its influence on the beam dynamics is very small.

\section{CENTRAL REGION}

3.1. Inflector Electrode Cut. The central region performance was investigated with intention to increase the beam transmission and quality. The following parameters were used in the calculations: RF frequency $31.02 \mathrm{MHz}$, ion source extraction voltage $22.3 \mathrm{kV}$, dee voltage $70 \mathrm{kV}$, inflector voltage $\pm 5.5 \mathrm{kV}$.

In the calculation below, the injected bunch consisted of 2000 particles with the upright transverse emittance $150 \pi \cdot \mathrm{mm} \cdot \mathrm{mrad}$ and longitudinal emittance $\pi \cdot 35^{\circ} \cdot 6 \mathrm{keV}$. Figure 9 shows the computer model of the central region with accelerated (grey points) and lost (black points) macroparticles.

In the TDB case the missing cut of the inflector electrodes at the entrance (Fig. 9) and $3^{\circ}$ cut at the exit (Fig. 10) are probably the cause for the difference between the calculated and theoretical central trajectory of the inflector. In the original inflector design, poor matching of the axial trajectory to the midplane of the cyclotron takes place, leading to axial oscillations $\sim 2 \mathrm{~mm}$. Since in the central region most particles get lost axially, the effect described above will substantially influence the beam transmission there. In the calculations it was shown that the

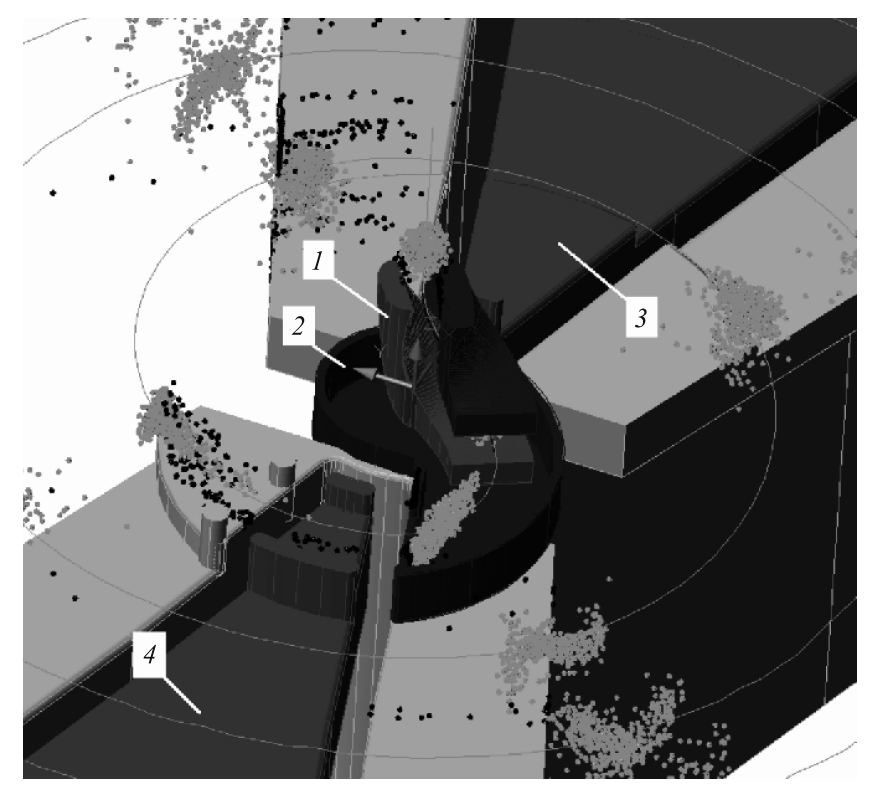

Fig. 9. Central region model: $1-$ inflector; $2-$ RF shield; $3-$ dee $2 ; 4-$ dee 1 


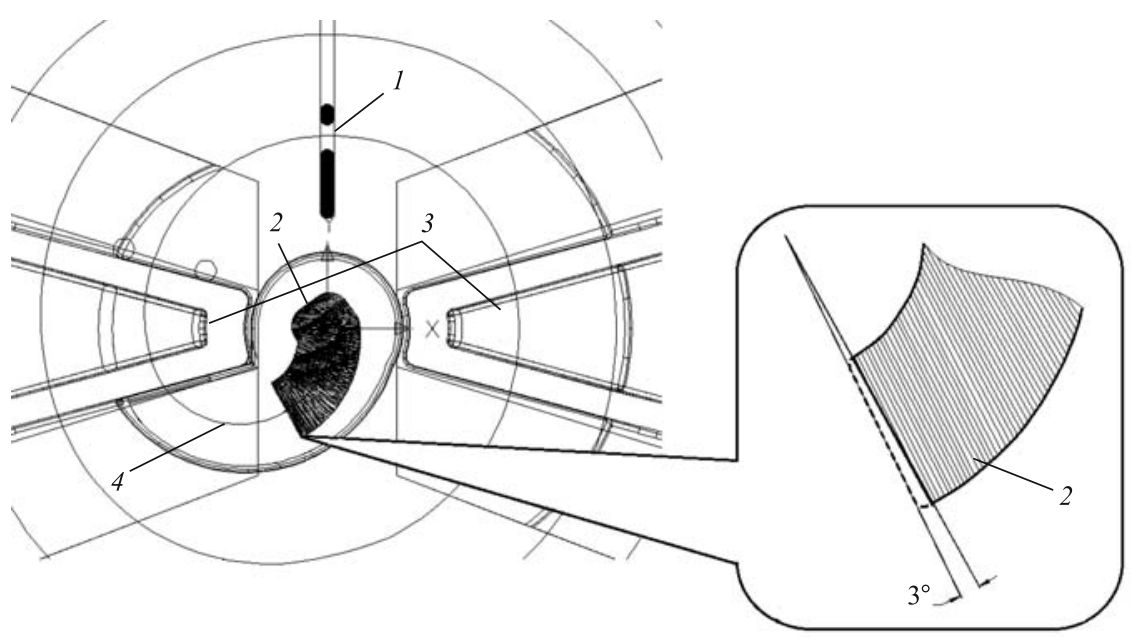

Fig. 10. Location of the phase slit with the inflector exit zooming: $1-$ phase slit; $2-$ inflector; 3 - dees; 4 - reference trajectory

best agreement of the calculated curve with the theoretical trajectory took place for the inflector entrance cut of $\sim 2-3 \mathrm{~mm}$ and for the exit cut of $\sim 7-8^{\circ}$. In this case axial oscillations reduce to $\sim 0.3 \mathrm{~mm}$ with the required inflector voltage slightly higher than the design value $5.5 \mathrm{kV}$. All the calculations below were performed with the original inflector, unless the inflector cut is mentioned explicitly.

3.2. Phase Slit. For the transmission from the buncher entrance to the 3rd cyclotron turn, both the bunching efficiency and CR transmission are taken
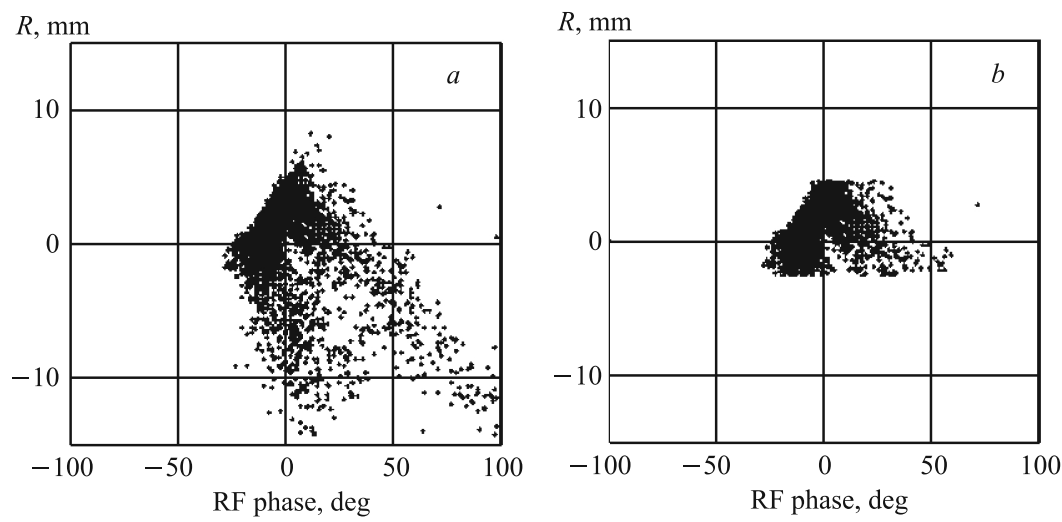

Fig. 11. Phase slit impact on the beam. There are beam projections in the place of the slit installation without the phase slit $(a)$ and with the slit $(b) ; R$ is the deviation of the ion radius from the reference value 

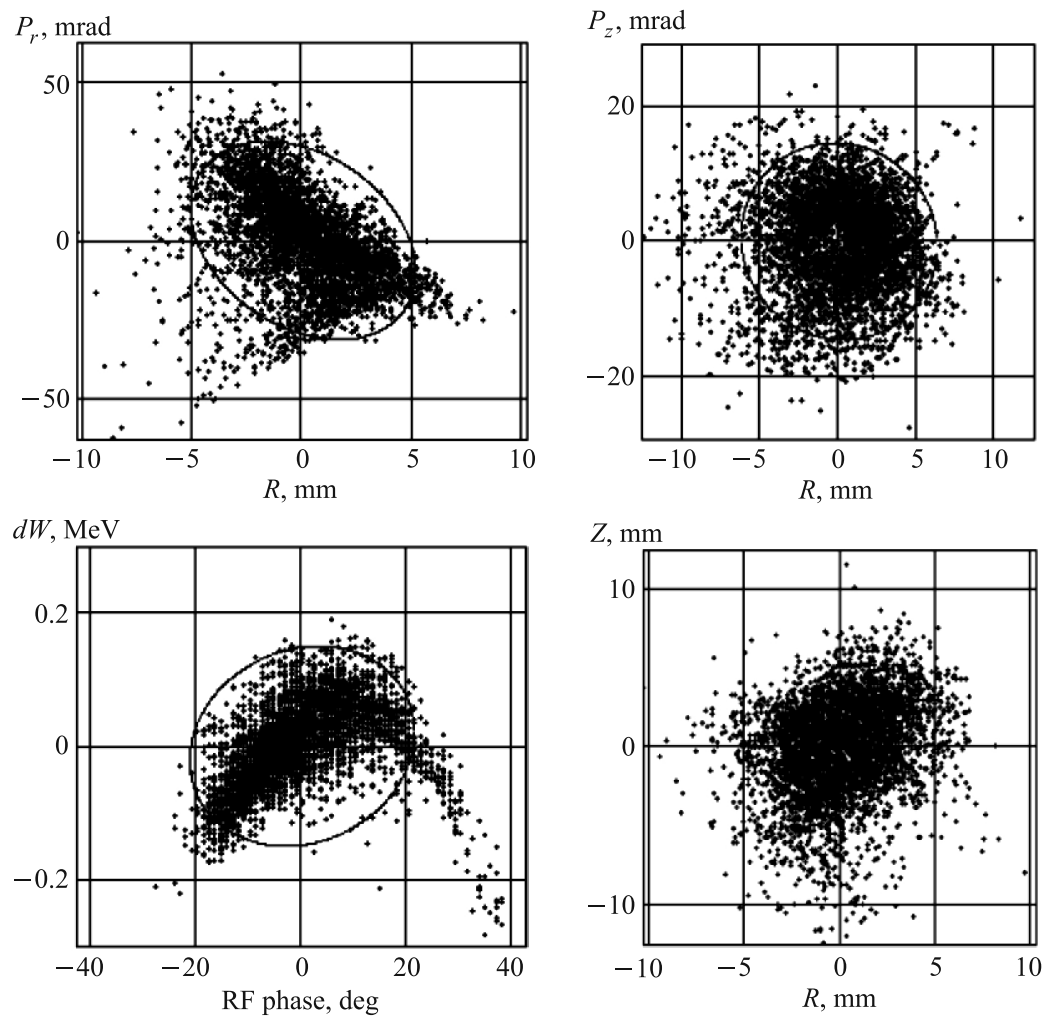

Fig. 12. Beam projections after completion of the 3 rd turn $\left(\theta=270^{\circ}\right)$ for the case with the phase slit: $P_{r}$ is the radial momentum divided by the total particle momentum; $R$ is the radius; $P_{z}$ is the axial momentum divided by the total particle momentum; $Z$ is the $Z$ coordinate; $d W$ is the deviation of the ion energy from the reference value. The solid line ellipses have half-axes equal to 2 standard deviations of the corresponding distributions

into account in the analysis. The correlated emittance and axial emittance are found to be rather large: 253 and $102 \pi \cdot \mathrm{mm} \cdot \mathrm{mrad}$. The bunch RF phase range is not small either: $2 \times 26^{\circ}$ in total. It is therefore suggested to install a phase slit to improve the beam quality in the central region.

The optimal position of the slit was selected downstream of the inflector exit at the 1st turn of the bunch in the cyclotron (Fig. 10). Calculations showed that the optimal radial slit gap was $7 \mathrm{~mm}$. The phase slit produced a factor of $\sim 3$ improvements in the RF phase width of the bunch: 2 standard RF phase deviations from the reference value $2 \sigma=48^{\circ} \mathrm{RF}$ at the entrance of the slit, and $2 \sigma=17^{\circ} \mathrm{RF}$ at the exit from the slit with only $11 \%$ reduction of the beam intensity (Fig. 11). 
Table 2. Beam quality improvement by the phase slit at the 3rd cyclotron turn

\begin{tabular}{|l|c|c|}
\hline \multicolumn{1}{|c|}{ Parameter } & No slit & With slit \\
\hline$d R, \mathrm{~mm}$ & 7 & 5 \\
\hline$P_{r}, \mathrm{mrad}$ & 35 & 31 \\
\hline$\varepsilon_{r}, \pi \cdot \mathrm{mm} \cdot \mathrm{mrad}$ & 253 & 162 \\
\hline$d Z, \mathrm{~mm}$ & 7 & 6 \\
\hline$P_{z}, \mathrm{mrad}$ & 16 & 15 \\
\hline$\varepsilon_{z}, \pi \cdot \mathrm{mm} \cdot \mathrm{mrad}$ & 102 & 91 \\
\hline$d W, \mathrm{MeV} / \mathrm{u}$ & 0.27 & 0.15 \\
\hline$d \phi,{ }^{\circ} \mathrm{RF}$ phase angle & 26 & 21 \\
\hline
\end{tabular}

The result of simulation of the ion tracing from the saw-tooth buncher entrance to the 3rd turn in the cyclotron with the initial emittance $75 \pi \cdot \mathrm{mm} \cdot \mathrm{mrad}$ is shown in Fig. 12.

The summary of the phase slit transformation of the beam can be found in Table 2. The following quantities are given in the table: axial $\left(\varepsilon_{z}\right)$ and radial $\left(\varepsilon_{r}\right)$ emittances, 2 standard deviations from the reference values of the radial $(d R)$ and axial $(d Z)$ displacements, radial $\left(P_{r}\right)$ and axial $\left(P_{z}\right)$ momenta, and energy $(d W)$ and RF phase $(d \phi)$ of ions. As the result of using the slit, at the 3rd cyclotron turn the beam radial emittance decreases by a factor of 1.6, energy spread by a factor of 1.8 , and RF phase range by a factor of 1.2 at the expense of the reduction of the overall beam transmission from the buncher entrance to the 3rd turn down to $48 \%$ from $52 \%$ for the case without the phase slit. So, installation of the phase slit is highly recommended for the cyclotron.

\section{BEAM EXTRACTION}

The design of the beam extraction system of the compact cyclotron came up with a layout [9] shown in Fig. 13. The extracted beam requirements for injection to the HITFiL are presented in [10]. The initial design of the extraction system has no focusing elements.

On the basis of the assessment of the magnetic and electrostatic fields of the extraction system elements, the extracted reference orbit of the beam was produced with the help of the substantially upgraded CBDA [11] code and newly developed SNOP code, see Fig. 4. Three-dimensional field distributions input in the codes ensures correct allowance for the impact of the nonlinear field effects on the beam dynamics [12].

4.1. Harmonic Coil. The calculations showed that the bunch RF phase angle becomes smaller by $\sim 15^{\circ}$ due to the azimuthally averaged magnetic field 
distortion by the harmonic coil field. The result can be explained by only one harmonic coil present in the system, and its contribution to the mean magnetic field is not compensated by another harmonic coil with the opposite excitation current that is normally used in the cyclotron [8]. As a result, the corresponding deviation of the mean magnetic field from the required isochronous dependence takes place, accompanied by the RF phase angle shift mentioned above. This fact could lead to an increase in the energy spread in the beam and some complication in the particle extraction. Also, there is some deviation in the radial betatron oscillation frequency, the beam dynamics effect of which should

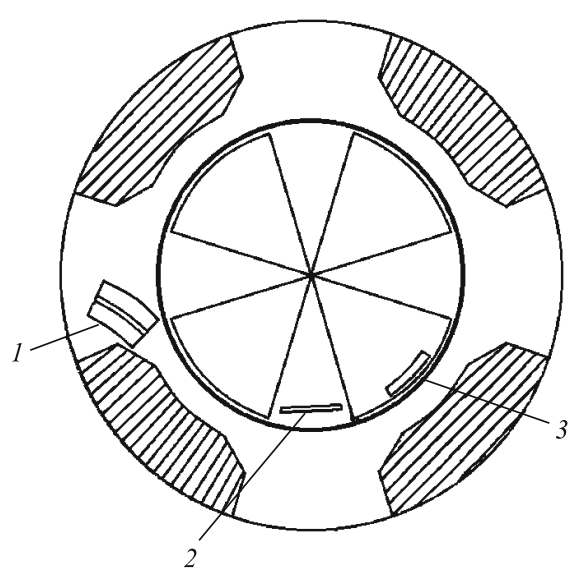

Fig. 13. Layout of the extraction system: 1 - magnetic channel; 2 - electrostatic deflector; 3 - harmonic coil still be estimated.

Concerning the efficiency of the only one harmonic coil for enlargement of the turn separation at the mouth of the electrostatic deflector (ES1), it works well when the beam acceleration is ideally centered. But in real life the off-centering of the order of $6 \mathrm{~mm}$ (our case) takes place, and the maximal turn separation does not occur at the ES1. As a result, the efficiency of the harmonic coil is not sufficiently good: sometimes just $\sim 2 \mathrm{~mm}$ gain in the turn separation was obtained with the harmonic coil switched on. The remedy would be centering of the beam as perfect as possible.

Another method would be installation of 2 pairs of harmonic coils azimuthally separated by $90^{\circ}$, to adjust the azimuth of the magnetic harmonic coil as needed in compliance with the existing «natural» beam off-centering. Since the harmonic coils come in pairs with the equal positive and negative contributions to the cyclotron field, no perturbation of the main magnetic field will occur. The RIKEN AVF Cyclotron HC is an example of such an arrangement [8].

There are essential restrictions connected with initial design of the structural elements of the cyclotron; therefore, it is impossible to add additional harmonic coils to this machine. Nevertheless, even with the only one harmonic coil the turn separation at the electrostatic deflector entrance is $11.8 \mathrm{~mm}$ and $6.5 \mathrm{~mm}$ without the harmonic coil. The result was obtained by optimization of the amplitude and position of the harmonic coils, but in experiments with the beam only one amplitude variation will be available with the harmonic coil position being fixed.

4.2. Electrostatic Deflector. The ES1 is located at $\sim 45^{\circ}$ downstream of the harmonic coil position (Fig. 13), which will provide not only sufficient turn 
separation between the neighboring turns but also a maximal positive angle of the extracted orbit in the direction of the ES1. As a result of the extraction studies, the eventually calculated optimal electrostatic deflector position to direct the beam to the magnetic channel (M2) entrance was somewhat readjusted as compared with the original (TDB) one in compliance with the best calculated cyclotron magnetic field (deviation from isochronous field less than $5 \mathrm{G}$ ).

The curvature of the ES1 septum requires adjustment to fit better to the extracted trajectory geometry. The suggestion was to change the original ES1 curvature radius from 6000 to $4500 \mathrm{~mm}$. The subsequent calculations showed that this change led to reduction of particle losses at the ES1.

The radial aperture of the ES1 in the TDB is constant and equals $8 \mathrm{~mm}$. This does not match the extracted beam dimensions, increasing along the ES1 length due to the radial defocusing of the beam in the cyclotron fringe field. In some cyclotrons [13] the ES1 consists of two sections with independent movement of the edges to regulate the effective septum curvature and thickness and the radial aperture of the ES1.

The TDB azimuthal length of the septum was greater than that of the potential electrode. The advantage of this feature for the beam dynamics is not clear. It causes additional losses of particles at the entrance of the deflector. The new ES1 design with the curvature radius changed from 6000 to $4500 \mathrm{~mm}$, equal septum and potential electrodes, and $10 \mathrm{~mm}$ radial aperture was adopted following the above recommendations.

The decrease in the main cyclotron magnetic field along the reference particle trajectory strongly defocuses the extracted beam in the horizontal plane in the location of the ES1 and downstream. To overcome the problem a transverse electrostatic gradient in the ES1 will help, but it is not applicable for this machine due to the required maximal design simplicity.

4.3. Gradient Corrector. To solve the problem of the strong horizontal defocusing of the beam downstream of the ES1 and at the entrance of the magnetic

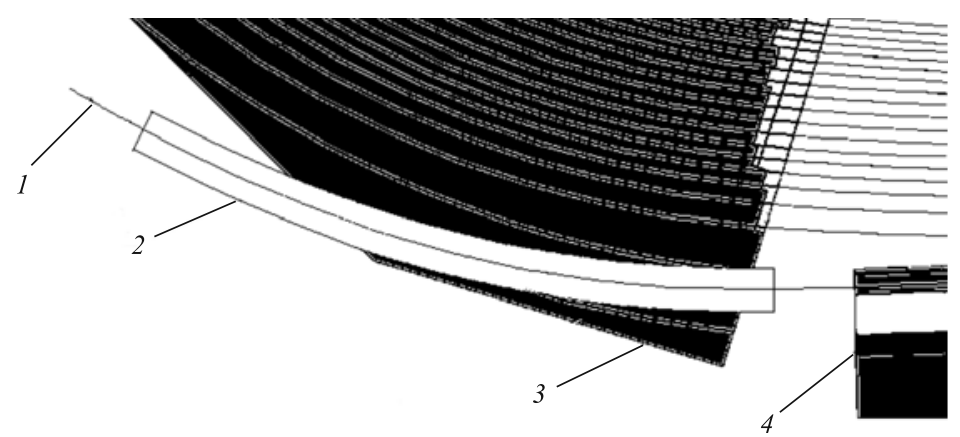

Fig. 14. The magnetic field region with the added focusing gradient: 1 - reference particle trajectory; 2 - gradient corrector region; 3 - magnet sector; 4 - electrostatic deflector 


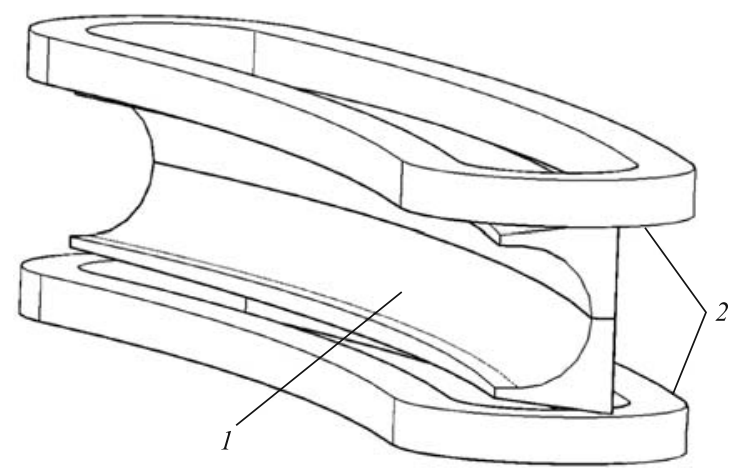

Fig. 15. Active gradient corrector: $1-$ iron; $2-$ coils

channel M2, an additional structure element, so-called gradient corrector (GC), was proposed, as in the RIKEN AVF Cyclotron [8]. A possible position of the GC is shown in Fig. 14. The calculations show that the optimal gradient to be added to the cyclotron field is $\sim 20 \mathrm{~T} / \mathrm{m}$. This value corresponds to the negative gradient of the main cyclotron field that ensures a good horizontal focusing. In this case the axial emittance is located in the 2nd and the 4th quadrants of the axial phase space at the entrance of the M2, which guarantees axial beam focusing downstream of the M2. With the GC, the beam quality and transmission were substantially improved at the IP position (100 mm downstream of the M2 exit) and at the exit of the BDS.

The preliminary calculations of the reference particle extraction in the latest version of the calculated magnetic field of the cyclotron showed that the deflecting power of the ES1 with the maximal design voltage $85 \mathrm{kV}$ [2] was not sufficient to direct the ion to the M2 channel mouth. This is a clear indication of the necessity to convert the gradient corrector into the gradient channel with the field drop approximately $-0.1 \mathrm{~T}$ to provide both the focusing and the necessary deflection of the beam.

As the result of the optimization study, an active gradient corrector (Fig. 15) emerged. The optimal position of the GC was adopted in compliance with the recommendations in Fig. 13.

4.4. Magnetic Channel. Transverse emittance distortion of the extracted beam by the nonlinear distribution of the M2 magnetic field normal to the ion orbit takes place. The calculated («Real B-field») magnetic field distribution in the midplane of the M2 magnet in the horizontal optical (local) coordinate system is given in Fig. 16. The field estimation was performed in the environment of the main cyclotron magnetic field. 


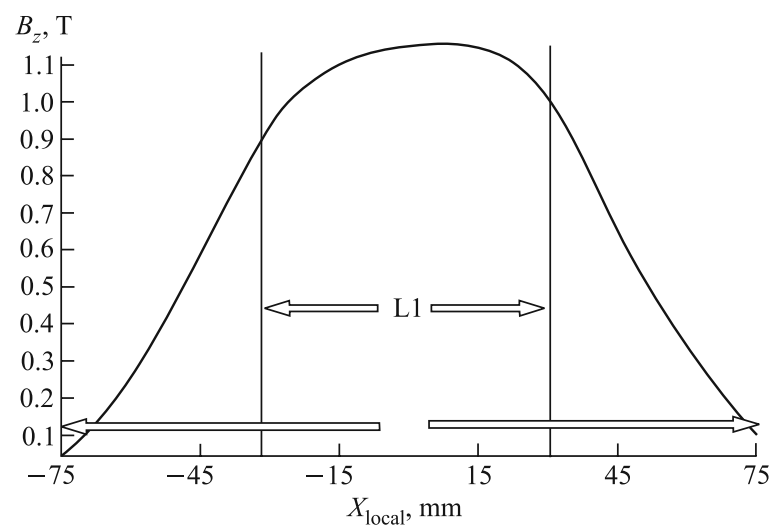

Fig. 16. M2 field plot along the line normal to the ion trajectory; L1 is the pole width, $\mathrm{L} 2$ is the distance between the horizontal yokes of the magnet, $Y_{\text {local }}=Z_{\text {local }}=0$

The induced aberrations in the beam optics could be corrected by appropriate shaping of the M2 magnetic field and introduction of the collimator. The M2 performance was analyzed for four cases:

1. M2 TDB structure distribution of the magnetic field in the environment of the main cyclotron field («Real B-field», Fig. 16).

2. Installation of the collimator to reduce the beam size in case 1 («Real B-field and Collimator»).

3. Uniform magnetic field of the M2 between the poles: constant $B_{z}$, and $B_{x}=B_{y}=0$ («Const B-field») with the collimator installed.

4. Analytical axial magnetic field of the M2 between the poles with the positive gradient of the field, $B_{x}=B_{y}=0$, and the collimator installed («B-gradient \& Collimator»).

The emittances at the IP position were looked at. In the simulation the beam was presented by 5000 particles at the ES1 entrance under the assumption of the one-turn extraction.

The effect of the collimator is reflected in the obtained emittances at the IP (Table 3). Analysis of the extracted beam quality including dispersion and its derivative was performed using the procedure described in [14].

To check the impact of the magnetic field nonlinearity, the real B-field distribution was replaced with the uniform one as described above. The results confirmed the expectation: the beam quality drastically improved.

Apparently, the next step would be applying the transverse focusing gradient in the M2 magnetic field distribution. For example, adding the $2 \mathrm{~T} / \mathrm{m}$ transverse gradient (decreasing field in the direction of the center of curvature of the M2 pole) to the M2 uniform field will substantially improve the beam quality. 
Table 3. Emittance $(\pi \cdot \mathbf{m m} \cdot \mathbf{m r a d})$ at the IP position

\begin{tabular}{|c|c|c|c|c|}
\hline & B-real & $\begin{array}{c}\text { B-real } \\
\& \\
\text { Collimator }\end{array}$ & $\begin{array}{c}\text { B-const } \\
\& \\
\text { Collimator }\end{array}$ & $\begin{array}{c}\text { B-gradient } \\
\& \\
\text { Collimator }\end{array}$ \\
\hline $\begin{array}{c}\text { Horizontal } \\
\text { emittance } \\
\text { Axial } \\
\text { emittance }\end{array}$ & 1400 & 243 & 81 & 7 \\
\hline
\end{tabular}

The preliminary estimation of the above-proposed magnetic field modification favors the M2 redesign. A new design of the so-called «combined-function» M2 magnetic channel was prepared. In the new design ampere-turns per coil were reduced to 17818 for the central field $B_{0}=1.1 \mathrm{~T}$ instead of 29232 in the TDB structure. The air gap was reduced to $30 \mathrm{~mm}$ instead of previous $40 \mathrm{~mm}$. The beam dynamics calculations show that this reduced gap is sufficient for the beam to pass through the magnet opening without extra ion losses. The reduced gap requirement was dictated by the necessity to ensure the linear field distribution transverse to the beam trajectory. The yoke became thinner; just what is needed to let the magnetic flux from the pole region pass through without the yoke got saturated. The coil cross section was reduced to keep about the same average current density as in the previous structure (same conductor current but reduced turn number). So, the total size of the magnet was decreased as compared with the TDB.

The new M2 pole profile was determined by the Opera2D [3] calculations with the cross-check by the Poisson-Superfish code [15]. The resulting shape of the pole is given in Table 4 in the local coordinates. The Opera3D simulation fully confirmed the result obtained in the middle cross section of the magnet.

Table 4. Magnetic channel air gap profile

\begin{tabular}{|c|c|c|c|c|c|c|c|}
\hline$X_{\text {local }}, \mathrm{mm}$ & \multicolumn{2}{|c|}{-30} & -25 & -20 & -15 & -10 & -5 \\
\hline Gap, mm & & .2 & 30.2 & 30.4 & 30.6 & 30.4 & 30.1 \\
\hline$X_{\text {loc }}$ & 0 & 5 & 10 & 15 & 20 & 25 & 30 \\
\hline Gap, mm & 30 & 29.7 & 29.3 & 28.7 & 28.4 & 28.2 & 28.2 \\
\hline
\end{tabular}

Calculations were made with allowance for the influence of the magnetic field of the cyclotron. Due to the main cyclotron field, the field level and the distribution along the ion trajectory are noticeably changed but the linearity of the field in the aperture $\pm 15 \mathrm{~mm}$ is not spoiled at all. For the analysis of the magnetic field linearity in the $\pm 15 \mathrm{~mm}$ transverse displacement from the reference orbit, a 
quadratic fit of the calculated field distribution was performed. The results are as follows: the field value in the center is $\sim 1.03 \mathrm{~T}$; the field transverse gradient is $\sim 2.06 \mathrm{~T} / \mathrm{m}$; the quadratic term of the distribution is $\sim 10^{-6} \mathrm{~T} / \mathrm{mm}^{2}$.

\section{CYCLOTRON PERFORMANCE IMPROVEMENT}

Some of the above-proposed changes to the Technical Design Baseline of the HITFiL injector cyclotron (inflector cuts, phase slit, HC position optimization, new ES1 structure, GC introduction, M2 redesign, etc.) were implemented and, as a result, the cyclotron performance drastically improved in terms of the beam transmission efficiency and the output beam quality.

5.1. Transmission. Estimation of the overall cyclotron transmission is a key issue of the machine design.

By summarizing the so far obtained results on the partial beam transmission through various units of the cyclotron (Fig. 17), the overall transmission could be assessed. There is an increase to approximately $13.1 \%$ in the transmission instead of $2.1 \%$ in the TDB case, although the output beam intensity increases less than that due to smaller ECR beam current adopted in this case.

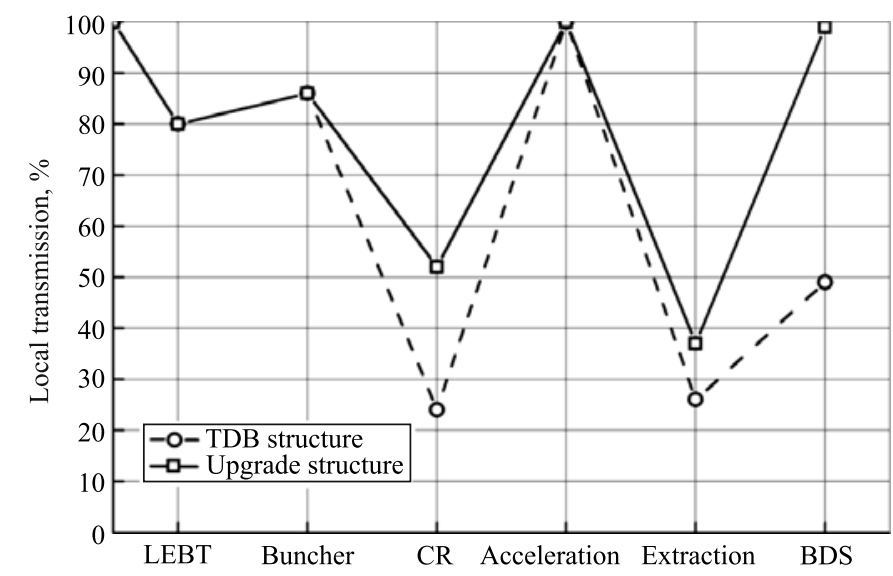

Fig. 17. Partial transmission (exit/entrance beam intensity ratio)

5.2. Beam Quality. Along with the estimation of the overall cyclotron transmission, the output beam quality is one of the basic requirements formulated before the design of the cyclotron begins. So, the output beam parameters (emittance, energy spread, time pulse, dispersion) are also the subject of the study.

The transformation of the $6 \mathrm{D}$ beam emittance in the external field was investigated with allowance for the ion space charge effects and particle losses at various limiting surfaces. 


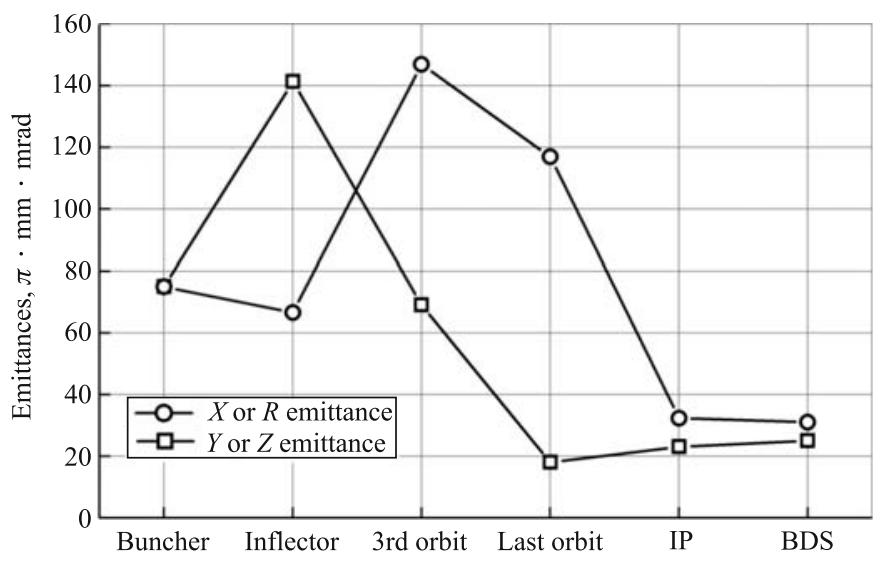

Fig. 18. Transverse emittance evolution

The summary of the beam emittance evolution is given in Fig. 18. The new ES1 structure described above, gradient corrector, and new M2 magnet design were assumed in the calculations.

Figure 18 shows that the axial RMS emittance became larger at the inflector exit due to the well-known effects of coupling of the transverse motions in the LEBT and an increase of the axial emittance in the inflector. The correlated radial RMS emittance increases downstream of the CR due to an increase in the energy spread in the beam during acceleration. The axial RMS emittance in this range decreases following adiabatic damping of the betatron oscillation. Reduction of this emittance at the last cyclotron orbit can be obtained by better matching of the beam in the LEBT to the cyclotron CR acceptance.

The energy spread in the bunch at the 1st HEBT lens entrance is lower than required $( \pm 1 \%)$. Momentum dispersion is $-1.506 \mathrm{~m}$, the derivative of dispersion is -0.304 there. At the HEBT entrance the transverse emittances with the collimator installed at the M2 are improved as compared with the TDB case but are still larger than the required ones. But the intensity, energy spread, and uncorrelated (local dispersion removed) radial emittance of the beam meet the requirements there.

To ensure the beam crossover (waist) at the HEBT entrance, additional elements could be installed in the BDS to match the beam at the IP position to the 1 st lens of the HEBT. The matching was produced by the Trace3D code [16] with the above calculated 6D beam emittance at the IP location as a starting point (Fig. 19). The quadrupole positions and gradients were parameters to determine samarium cobalt $(\mathrm{SmCo})$ or neodymium iron boron $(\mathrm{NdFeB})$ can be selected for the Permanent Magnet Quadrupole (PMQ) with the design gradient of $10 \mathrm{~T} / \mathrm{m}$, 


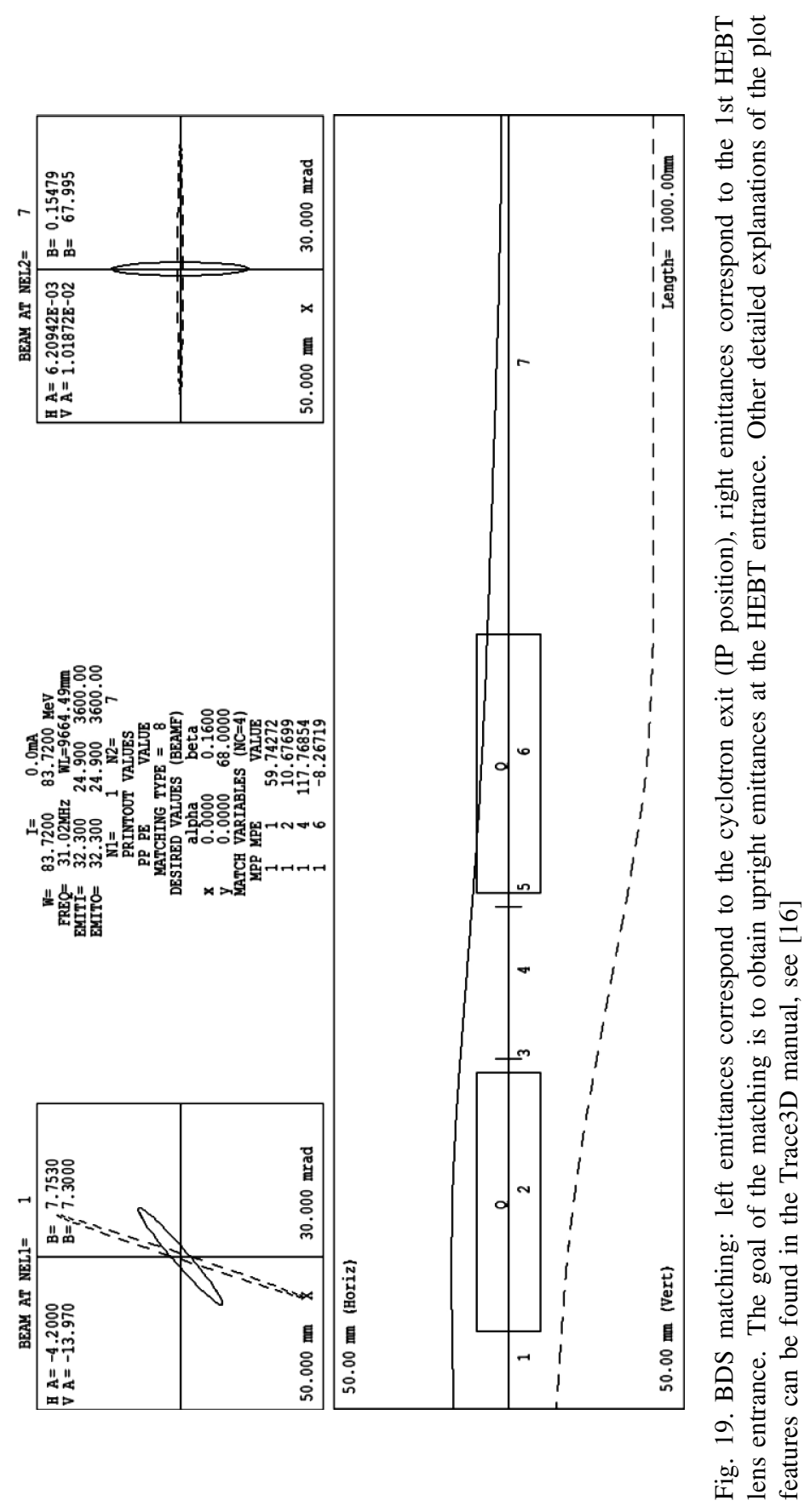


aperture $100 \mathrm{~mm}$, and length $200 \mathrm{~mm}$. The field at the quadrupole pole tip is $0.5 \mathrm{~T}$, which is feasible, and the relevant devices are now commercially available. The variable-strength Halbach-type PMQ will do in this case.

\section{CONCLUSIONS}

The HITFiL injector cyclotron beam property was simulated starting from the buncher entrance to the 1st HEBT lens.

Some of the proposed upgrades to the Technical Design Baseline of the cyclotron were implemented, which led to substantial improvement of the previous design.

A drastic increase in the transmission (factor of 5) takes place, although the output beam intensity increases less due to smaller ECR beam current adopted in these calculations.

In the TDB design of the cyclotron there were no focusing elements in the extraction system except rather weak edge focusing in the magnetic channel M2. This led to complete horizontal divergence of the output beam and axial overfocusing due to the sharp drop of the main cyclotron magnetic field.

Introduction of the focusing elements permits decent transverse RMS emittances and required energy spread at the exit of the cyclotron, although the transverse emittances are still larger than the required ones, and some improvements in this direction are expected. For example, the central region revision would probably improve the axial quality of the beam at extraction.

The change of the magnetic field structure with the air gap between the sectors increased to $80 \mathrm{~mm}$ (conceptual modification!) prevents crossing of dangerous resonances during ion acceleration.

Acknowledgements. The authors are grateful to Prof. Yuan Youjin for detailed discussions and important suggestions as to the subject of the study, Dr. Zhang ShengHu for consultations on the RF system design of the machine, Dr. Shi AiMing for providing us with the initial design of the saw-tooth buncher, and Dr. Alexey Vorozhtsov for helping us with the M2 channel design and selection of the PMQs for the BDS.

\section{REFERENCES}

1. Zhang J. Q., Song M.T., Wei B. W. Design of Synchrotron for Hadron Therapy // High Energy Physics and Nuclear Physics. 2007. V.31. P. 1122-1125.

2. Wang B. et al. Design and Construction Progress of a $7 \mathrm{MeV} / \mathrm{u}$ Cyclotron // Proc. 19th Int. Conf. on Cyclotrons and Their Applications, 2010.

3. OPERA/TOSCA Reference Manual. Vector Fields Limited. Oxford, OX5 1JE, England. 
4. Gordon M.M., Welton T.A. Oak Ridge National Lab, ORNL Rpt. No. 2765, 1959.

5. Grigoriev Yu.N., Vshivkov V.A., Fedoruk M.P. Numerical Particle-in-Cell. Theory and Applications, VSP BP. Utrecht, Boston, 2002.

6. Zhang J. et al. Axial Injection Beam Line of a Compact Cyclotron // Proc. 19th Int. Conf. on Cyclotrons and Their Applications, 2010.

7. Facility for Rare Isotope Beams (FRIB). Conceptual Design Report. Michigan State University, MI, USA, July 2010. P. (6-29)-(6-30).

8. Vorozhtsov S.B. et al. Calculations of the Beam Transmission and Quality in the RIKEN AVF Cyclotron // Proc. 21st Russian Accelerator Conference, 2008. P. 51-53.

9. Hao H.F. et al. Beam Extraction System of Compact Cyclotron // Proc. 19th Int. Conf. on Cyclotrons and Their Applications, 2010.

10. Zhang J.Q., Song M.T., Wei B.W. Injection System Design for a Hadron Therapy Synchrotron // Chinese Physics C. 2008. V.32(05): 393396 ISSN: 1674-1137 CN: 11-5641/O4.

11. Perepelkin E. E., Vorozhtsov S. B. CBDA - Cyclotron Beam Dynamics Analysis Code // Proc. 21st Russian Accelerator Conference, 2008. P. 40-42.

12. Milton B. F. A Cyclotron Orbit Code Using Second Order Transfer Matrices // Proc. 11th Int. Conf. on Cyclotrons and Their Applications, 1987. P. 240-243.

13. Bakewicz E. et al. A New Extraction System for the Upgraded AIC-144 Cyclotron // Nukleonika. 2001. V.46, No. 2. P.51-57.

14. Vorozhtsov S. B., Perepelkin E.E., Onischenko L.M. Customs Cyclotron and Beam Delivery System // Proc. 18th Int. Conf. on Cyclotrons and Their Applications, 2007. P. 421-423.

15. Halbach K., Holsinger R. F. SUPERFISH - A Computer Program for Evaluation of RF Cavities with Cylindrical Symmetry // Particle Accelerators. 1976. V.7. P. 213222.

16. Crandall K. R., Rusthoi D. P. TRACE 3-D Documentation, LA-UR-97-886.

Received on August 3, 2011. 


\section{Редактор Е.И. Кравченко}

Подписано в печать 26.10.2011.

Формат $60 \times 90 / 16$. Бумага офсетная. Печать офсетная.

Усл. печ. л. 1,44. Уч.-изд. л. 2,00. Тираж 240 экз. Заказ № 57467.

Издательский отдел Объединенного института ядерных исследований 141980, г. Дубна, Московская обл., ул. Жолио-Кюри, 6.

E-mail: publish@jinr.ru www.jinr.ru/publish/ 